CONFORMAL GEOMETRY AND DYNAMICS

An Electronic Journal of the American Mathematical Society

Volume 5, Pages 140-152 (October 18, 2001)

S $1088-4173(01) 00070-4$

\title{
CONTINUITY OF HAUSDORFF DIMENSION OF JULIA-LAVAURS SETS AS A FUNCTION OF THE PHASE
}

\author{
MARIUSZ URBANSKI AND MICHEL ZINSMEISTER
}

\begin{abstract}
Let $f_{0}(z)=z^{2}+1 / 4$ and $\mathcal{E}_{0}$ the set of phases $\bar{\sigma}$ such that the critical point 0 escapes in one step by the Lavaurs map $g_{\sigma}$; it is a topological strip in the cylinder of phases whose boundary consists of two Jordan curves symmetric wrt $\mathbb{R} / \mathbb{Z}$. We prove that if $\bar{\sigma}_{n} \in \mathcal{E}_{0}$ converges to $\bar{\sigma} \in \partial \mathcal{E}_{0}$ in such a way that $g_{\sigma_{n}}(0)$ converges to $g_{\sigma}(0)$ along an external ray, then the Hausdorff dimension of the Julia-Lavaurs set $J\left(f_{0}, g_{\sigma_{n}}\right)$ converges to the Hausdorff dimension of $J\left(f_{0}, g_{\sigma}\right)$.
\end{abstract}

\section{IntroduCtion}

Let $\mathbb{C}_{q}[z] \simeq \mathbb{C}_{q}$ be the set of monic polynomials of degree $q \geq 2$. For every $f \in \mathbb{C}_{q}[z]$ one defines the filled - in Julia set $K(f)$ as the set of points $z$ such that the sequence defined inductively by $z_{0}=z, z_{n+1}=f\left(z_{n}\right)$ does not converge to $\infty$. It is a non-empty compact set whose boundary, denoted by $J(f)$ is called the Julia set of $f$. The Julia set is the chaotic locus of the dynamics of $f$ in the sense that it is the set of points of non-normality for the family of iterates of $f$. It is, except for a few exceptions, a fractal set and this makes $d(f)$, the Hausdorff dimension of $J(f)$, a relevant quantity to study.

Our paper deals with the function $f \longmapsto d(f), \mathbb{C}_{q}[z] \longrightarrow \mathbb{R}$. This function is of course strongly related to the "topological" function $f \longmapsto J(f), \mathbb{C}_{q}[z] \longrightarrow$ $\mathcal{K}(\mathbb{C})$, where $\mathcal{K}(\mathbb{C})$ stands for the set of non-empty compact subsets of the plane, equipped with the Hausdorff metric. Douady [1] has proven that if $f_{0} \in \mathbb{C}_{q}[z]$ has no indifferent periodic point which is either rational or linearizable, then the mapping $f \longmapsto J(f)$ is continuous at $f_{0}$. In particular, $J$ is continuous on the neighborhood of every "hyperbolic" $f_{0}$, that is if all critical points of $f_{0}$ are attracted by attracting cycles. In fact much more is true in this case: $J(f)$ moves in a holomorphic motion in the sense of [1]. Concerning the function $d$, Ruelle [10] has shown that it is real-analytic around every hyperbolic polynomial. Thus, as is usual in dynamical systems, topological changes or discontinuities appear at "points" $f_{0}$ having an indifferent cycle, a set containing as a dense subset polynomials having a parabolic cycle. Since this phenomenon may be extremely complicated, in order to study the nature of the discontinuities that occur, we restrict our attention to quadratic polynomials, i.e., to the one-parameter family $f(z)=z^{2}+c, c \in \mathbb{C}$. The set of

Received by the editors September 18, 2000 and, in revised form, June 28, 2001.

2000 Mathematics Subject Classification. Primary 37F45; Secondary 37F35, 37F15.

The research of the first author was partially supported by the NSF Grant DMS 9801583. He wishes to thank the University of Orleans and IHES, where a part of the research was done, for warm hospitality and excellent working conditions. 
bifurcation points is then a dense subset of the boundary of the Mandelbrot set and we focus on the "simplest possible" point, namely the polynomial $f_{0}(z)=z^{2}+\frac{1}{4}$ for which $1 / 2$ is a fixed point with multiplier 1 . We first restrict ourselves even further: to real values of $c$. By the preceeding discussion, the function $c \longmapsto d(c)=$ $d\left(z^{2}+c\right)$ is real-analytic on $[0,1 / 4) \cup(1 / 4,+\infty)$ and it is not difficult to see that the topological function is continuous from the left at $1 / 4$. In [7] it is proven that the function $d$ is also left-continuous at $1 / 4$, with a vertical tangent 9 . Douady [1] has shown that the topological function is discontinuous from the right at $1 / 4$. Later it was shown in [6] that the dimension function is also right-discontinuous. More precisely it is proven in [6] that

$$
d(1 / 4)<\liminf _{c \longrightarrow \frac{1}{4}+0} d(c) \leq \limsup _{c \longrightarrow \frac{1}{4}+0} d(c)<2 .
$$

Douady's result is much more precise: there is a whole circle of limit points for the topological function at $\frac{1}{4}+0$. The corresponding result for the dimension function is unknown: more specifically, can the $\leq$ sign be replaced by $<$ in (1.1)? This question was raised by Douady. Even if it does not solve this problem, the present paper aims to shed some light on it. In order to state precisely the result, we first need to recall some facts from parabolic implosion, the phenomenon which is responsible for discontinuities. Then we briefly touch on some selected facts from the theory of infinite conformal IFS in the sense of [4] which will be needed in the proof of the main theorem. We would like to add that in fact the Julia-Lavaurs sets appear to be a mine of infinite IFS (see [6]) and our explorations go beyond the [6] case in the sense that the IFS structure is obtained here as a refining of the construction presented there.

\section{A QUICK OVERVIEW OF PARABOLIC IMPLOSION}

(a) Fatou coordinates. We recall that $f_{0}(z)=z^{2}+\frac{1}{4}$. If $x \in \mathbb{C}$ and $r>0$, then $B(x, r)$ denotes the open ball with the center at $x$ and with radius $r$. If $\sigma \in \mathbb{C}$, we denote by $T_{\sigma}$ the translation $z \longmapsto z+\sigma$. We will also denote by $\pi$ the canonical projection from $\mathbb{C}$ onto $\mathbb{C} / \mathbb{Z}$. The inversion

$$
z \longmapsto Z=-\frac{1}{z-\frac{1}{2}}
$$

conjugates $f_{0}$ to the mapping

$$
F_{0}(Z)=Z+1+\frac{1}{Z-1}
$$

which is very close to the translation $T_{1}$ in the two domains $U_{M}^{-}=\{\operatorname{Re} z<-M\}$, $U_{M}^{+}=\{\operatorname{Re} z>M\}$, for large $M$. This conjugation may actually be modified to yield an exact conjugation to $T_{1}$ on $U_{M}^{ \pm}$. Returning to the variable $z$ we have more precisely:

Theorem 2.1. There exist holomorphic injective maps

$$
\varphi^{-}: V^{-}=B(3 / 8,1 / 8) \longrightarrow \mathbb{C}, \varphi^{+}: V^{+}=B(5 / 8,1 / 8) \longrightarrow \mathbb{C}
$$

such that $\varphi^{-}\left(V^{-}\right) \supset U_{M}^{-}, \varphi^{+}\left(V^{+}\right) \supset U_{M}^{+}$for some $M>0$ and such that

(i) $f_{0}$ is injective in $V^{-}, f_{0}\left(V^{-}\right) \subset V^{-}$and $\varphi^{-}\left(f_{0}(z)\right)=\varphi^{-}(z)+1, z \in V^{-}$,

(ii) $f_{0}$ is injective in $V^{+}, V^{+} \subset f_{0}\left(V^{+}\right)$and $\varphi^{+}\left(f_{0}(z)\right)=\varphi^{+}(z)+1$ if $z, f_{0}(z) \in V^{+}$. 
Moreover $\varphi^{ \pm}$are unique up to additive constant. They are called the Fatou coordinates (attracting and repelling). We will denote by $\Phi^{ \pm}$the expression of the Fatou coordinates in the $Z$-variable.

The mapping $\Phi^{+}$extends as an injective holomorphic function to the domain $\{y>x+M\} \cup\{y<-x-M\}$ and similarly $\Phi^{+}$to the domain symmetric with respect to the imaginary axis. Consequently the function $h=\Phi^{-} \circ\left(\Phi^{+}\right)^{-1}=\varphi^{-} \circ\left(\varphi^{+}\right)^{-1}$ is well defined in $\{y>|x+M|\} \cup\{y<-|x+M|\}$. Since $h(Z+1)=h(Z)+1$ whenever both $Z$ and $Z+1$ belong to the domain of $h$, we can define two maps $h^{\downarrow}, h^{\uparrow}$ holomorphic in $\{y>M+1\},\{y<-M-1\}$ respectively, "fixing" the point at $\infty$, and satisfying $h^{\downarrow \uparrow}(Z+1)=h^{\downarrow \uparrow}(Z)+1$. These maps are the horn maps associated to $f_{0}$.

Finally it can be shown that $\varphi^{-}$has an extension to $K\left(f_{0}\right)$ as a holomorphic function still satisfying the functional relation in (i), while $\Psi^{+}=\left(\varphi^{+}\right)^{-1}$, a priori only defined on $U_{M}^{+}$, can be extended as an entire function satisfying $\Psi^{+}(Z+1)=$ $f_{0}\left(\Psi^{+}(Z)\right)$. These two functions are called extended Fatou coordinates.

\section{(b) Lavaurs maps.}

Definition 2.2. A Lavaurs map for $f_{0}$ is a map from $K\left(f_{0}\right)$ to $\mathbb{C}$ of the form

$$
g_{\sigma}=\Psi^{+} \circ T_{\sigma} \circ \varphi^{-} .
$$

It follows easily from properties of the Fatou coordinates that

$$
g_{\sigma} \circ f_{0}=f_{0} \circ g_{\sigma}=g_{\sigma+1} .
$$

The "raison d'etre" of the preceeding definition is the following important theorem of Douady:

Theorem 2.3. Let $\epsilon_{n}$ be a sequence of complex numbers with positive real part converging to 0 in such a way that there exists a sequence of integers $N_{n} \longrightarrow+\infty$ such that

$$
-\frac{\pi}{\sqrt{\epsilon_{n}}}+N_{n} \longrightarrow \sigma \in \mathbb{C}
$$

(the square root is the one with positive real part), then, if

$$
f_{\epsilon}(z)=z^{2}+\frac{1}{4}+\epsilon
$$

$f_{\epsilon_{n}}^{N_{n}}$ converges uniformly on compact subsets of $K\left(f_{0}\right)$ towards the Lavaurs map $g_{\sigma}$.

(c) Dynamics of $\left(f_{0}, g_{\sigma}\right)$. For $k \geq 0, l \geq 0$, we define

$$
g_{\sigma}^{k, l}=f_{0}^{l} \circ g_{\sigma}^{k}
$$

on some domain depending on $k$ and $\sigma$, while if $k \geq 1, l<0$, there exists a domain depending on $k$ and $\sigma$ on which one can define

$$
g_{\sigma}^{k, l}=g_{\sigma+l} \circ g_{\sigma}^{k-1} .
$$

We call the pair $(k, l)$ admissible if $k=0, l \geq 0$ or if $k \geq 1, l \in \mathbb{Z}$. We (totally) order the set of admissible pairs by lexicographic order, and the usual vocabulary of iteration extends to this setting. The situation is only slightly complicated by the fact that the maps $g_{\sigma}^{k, l}$ are not everywhere defined. This leads to the following.

Definition 2.4. A point $z \in \mathbb{C}$ is said to escape by $\left(f_{0}, g_{\sigma}\right)$ if there exists $m \geq 0$ such that $g_{\sigma}^{m}(z)$ is well defined but belongs to $\mathbb{C} \backslash K\left(f_{0}\right)$ (in particular points in $\mathbb{C} \backslash K\left(f_{0}\right)$ escape, simply take $\left.m=0\right)$. 
Definition 2.5. The filled-in Julia-Lavaurs set $K\left(f_{0}, g_{\sigma}\right)$ is the set of non-escaping points. It is a compact set with

$$
J\left(f_{0}\right) \subset K\left(f_{0}, g_{\sigma}\right) \subset K\left(f_{0}\right) .
$$

The Julia-Lavaurs set $J\left(f_{0}, g_{\sigma}\right)$ is the boundary of $K\left(f_{0}, g_{\sigma}\right)$.

By the properties of the dynamics of $\left(f_{0}, g_{\sigma}\right)$, these sets depend only on $\pi(\sigma)$. Douady [1] has shown that $J\left(f_{0}, g_{\sigma}\right)$ is the closure of the set of points $z$ for which there exists $m \geq 0$ such that $g_{\sigma}^{m}(z)$ is well-defined and belongs to $J\left(f_{0}\right)$. It is also true that $J\left(f_{0}, g_{\sigma}\right)$ is the closure of the set of periodic repelling points; the proof for the classical case goes through without changes.

(d) Fatou-Julia-Lavaurs-Sullivan classification. Lavaurs in [1] has extended to this new dynamics the Fatou-Julia-Sullivan classification of components of the Fatou set. He has extended Sullivan's non-wandering theorem, proving that every component of $K\left(f_{0}\right)$ is eventually periodic in the sense of the new dynamics. Classical examples of periodic components have their counterpart in the new setting: attracting or parabolic basins, Siegel disks. However, there are new examples of periodic components. In order to observe them, we notice that $\Phi^{-}$conjugates the Lavaurs map $G_{\sigma}$ into the two horn maps $h_{\sigma}^{\downarrow \uparrow}=h^{\downarrow \uparrow} o T_{\sigma}$ and this conjugation holds in the domains $\{y>M\},\{y<-M\}$ for large enough $M$. Using a change of variable $u=\exp ( \pm 2 i \pi Z)$, the horn map is transferred to the germ of a holomorphic function fixing 0 . It thus makes sense to say that $\downarrow \uparrow \infty$ is either attracting, repelling or indifferent and if one of the ends is either attracting or parabolic or linearizable irrationally indifferent, it leads to periodic components of $K\left(f_{0}, g_{\sigma}\right)$ containing the parabolic fixed point $1 / 2$ on its boundary. We say in this case that $1 / 2$ is virtually attracting, parabolic or linearizable at $\downarrow \infty$ or $\uparrow \infty$ and the corresponding basin is called virtual. Lavaurs has shown in [1] that all periodic components of $K\left(f_{0}\right)$ are of one of the types just described.

(e) Limiting shapes of Julia sets. We may now state (recall that $f_{\epsilon}(z)=$ $\left.z^{2}+\frac{1}{4}+\epsilon\right)$ the following.

Theorem 2.6 (Douady [1], Lavaurs [2]). If $\sigma \in \mathbb{C}$ is such that $\left(f_{0}, g_{\sigma}\right)$ has no parabolic cycle, is not a linearizable irrationally indifferent cycle (including virtual components) and if $\epsilon_{n}$ converges to 0 as in Theorem 2.3, then $J\left(f_{\epsilon_{n}}\right) \longrightarrow J\left(f_{0}, g_{\sigma}\right)$ in the Hausdorff metric. Furthermore,

$$
J\left(f_{0}\right) \varsubsetneqq J\left(f_{0}, g_{\sigma}\right) \varsubsetneqq K\left(f_{0}, g_{s} g\right) .
$$

The phenomenon described by this theorem is called parabolic implosion. Actually, if $\sigma$ is an exceptional point in Theorem 2.6 then $J\left(f_{0}, g_{\sigma}\right)$ is still a limiting point of some sequence $J\left(f_{\epsilon_{n}}\right)$ but one must specify the way $\epsilon_{n}$ converges to 0 . If one is not careful enough, then a new parabolic implosion occurs, with new phase space, and this makes the set of limiting points very complicated to describe.

(f) The phase space. As in the classical study of the parameter space for the quadratic family, one can define a parameter space for the dynamics of $\left(f_{0}, g_{\sigma}\right)$. We denote by $\mathcal{E}$ the set of $\sigma \in \mathbb{C}$ such that the critical point 0 escapes by $\left(f_{0}, g_{\sigma}\right)$ and by $\mathcal{M}$ (for Mandelbrot) the complement of $\mathcal{E}$. Since the dynamics of $\left(f_{0}, g_{\sigma}\right)$ really depend only on $\pi(\sigma)$, one defines the phase space as $\mathbb{C} / \mathbb{Z}$ divided into $\pi(\mathcal{E})$ and $\pi(\mathcal{M})$. If one transforms the parameter plane of $c^{\prime} \mathrm{s}$ by the mapping 
$\theta: c \longmapsto-\frac{\pi}{c-1 / 4}$ and define $M_{n}=\theta(M) \cap[-(n+1),-n] \times \mathbb{R}$, then it is conjectured that $\pi\left(M_{n}\right)$ converges in the Hausdorff topology to some translate of $\pi(\mathcal{M})$. If the Fatou coordinates have been chosen so that they preserve the real axis, one can show that $\mathcal{M} \supset\{y \geq \pi\} \cup\{y \leq-\pi\}$, the union of two half-planes corresponding to the parabolic fixed point being attracting or indifferent at the corresponding end (and this corresponds by the mapping $\theta$ to the main cardioid). The set $\mathcal{E}$ is the union of the $\mathcal{E}_{n}^{\prime} s, n \geq 0$, where

$$
\mathcal{E}_{n}=\left\{\sigma \in \mathbb{C} ; g_{\sigma}^{n+1}(0) \in \mathbb{C} \backslash K\left(f_{0}\right)\right\} .
$$

With the above choice of Fatou coordinates we have $\mathbb{R} \subset \mathcal{E}_{0}$ and we may describe the whole component $\mathcal{E}_{0}$ as follows: From the formula defining $g_{\sigma}$ it appears that

$$
\mathcal{E}_{0}=\left(\Psi^{+}\right)^{-1}\left(\mathbb{C} \backslash K\left(f_{0}\right)\right) .
$$

This is a one-periodic strip bounded by two Jordan curves that are symmetric with respect to the real axis. If $\sigma \in \mathcal{E}_{0}$, then, since the critical point escapes by $g_{\sigma}$, one cannot have a parabolic point nor a Siegel disk; it follows that Theorem 2.6 applies for points in $\mathcal{E}_{0}$. Moreover, the method of [6] goes through in this case; the Hausdorff dimension of $J\left(f_{\epsilon_{n}}\right)$ converges to the Hausdorff dimension $d(\sigma)$ of $J\left(f_{0}, g_{\sigma}\right)$ if $\epsilon_{n} \longrightarrow 0$ as in Theorem 2.6.

As a natural extension of Douady's question, one can ask the following: is $d(\sigma)$ constant on $\mathcal{E}_{0}$ ? A further argument for the pertinence of this question is the twin paper [3], where it is proved that $d(\sigma)$ is real-analytic in $\mathcal{E}_{0}$.

Since the function $d$ is also sub-harmonic in $\mathcal{E}_{0}$, it is natural to study this question, to investigate the boundary behavior of the function in $\mathcal{E}_{0}$.

We define the radial approach to $\bar{\sigma} \in \pi\left(\partial \mathcal{E}_{0}\right)$ as the approach along the curve $\pi\left(\varphi^{+}\left(\Gamma_{z}\right)\right)$ where $z \in \partial K\left(f_{0}\right) \cap B\left(\frac{5}{8}, \frac{1}{8}\right), \Gamma_{z}$ is the external ray landing at $z$ and $\bar{\sigma}=\pi\left(\varphi^{+}(z)\right)$.

The main result of this work is the following:

Theorem 2.7. The function $d$ is radially continuous at every point of $\left.\partial \pi\left(\mathcal{E}_{0}\right)\right)$. Moreover, $d(\bar{\sigma})>\frac{4}{3}$ if $\bar{\sigma}=\pi\left(\varphi^{+}(z)\right)$ where $z$ is a preparabolic point for $f_{0}$ (i.e., $f_{0}^{n}(z)=\frac{1}{4}$ for some $\left.n \geq 0\right)$.

(g) Phase dependence of Julia-Lavaurs sets. In this section we prove the following result which is the topological ingredient needed to prove Theorem 2.7

Proposition 2.8. The function $\sigma \longmapsto J\left(f_{0}, g_{\sigma}\right)$ is continuous from $\overline{\mathcal{E}}_{0}$ to $\mathcal{K}(\mathbb{C})$.

Indeed, Douady's results from [1] extend without changes to the Julia-Lavaurs situation. In particular Proposition 2.8 follows from the following:

Proposition 2.9. If $\sigma \in \overline{\mathcal{E}}_{0}$, then $K\left(f_{0}, g_{\sigma}\right)=J\left(f_{0}, g_{\sigma}\right)$.

Proof. By the preceeding overview of parabolic implosion, in order to prove Proposition 2.9] it is sufficient to demonstrate that all periodic points are repelling except for the parabolic fixed point $1 / 2$ which is virtually repelling at both ends if $\sigma \in \overline{\mathcal{E}}_{0}$. The fact that the parabolic point is virtually repelling at both ends follows from the fact that $\overline{\mathcal{E}}_{0}$ lies entirely inside the strip $\{|y|<\pi\}$ while $\sigma$ 's with virtual indifferent or attracting ends belong to $\{|y| \geq \pi\}$. To prove that all periodic points are repelling, one simply observes that the critical orbit (under $\left(f_{0}, g_{\sigma}\right)$ ) accumulates only on $J\left(f_{0}\right)$. Let $z$ be a periodic point: if $z \in J\left(f_{0}\right)$, then it is a periodic point of $f_{0}$ and thus it is repelling or equal to $1 / 2$. If $z \in U_{0}=\operatorname{Int}\left(K\left(f_{0}\right)\right)$, it is then fixed 
by some $G=g_{\sigma}^{k, l}$ and $z \in W=U_{0} \backslash \bar{P}$, where $P$ denotes the critical orbit under $\left(f_{0}, g_{\sigma}\right)$. However, one can then build an inverse map of $G$ from the universal cover of $W$ into itself fixing $z$. By Schwarz's lemma, this point must be attracting and thus $z$ is repelling for $G$.

\section{A QUICK OVERVIEW OF CONFORMAL INFINITE IFS}

Let $S=\left\{\phi_{i}: X \rightarrow X\right\}_{i \in I}, X \subset \mathbb{R}^{d}$ for some $d \geq 1$, be a conformal (infinite) iterated function system (abbreviated as IFS) in the sense of [4]. For every $n \geq 1$ and $\omega \in I^{n}$, put

$$
\phi_{\omega}=\phi_{\omega_{1}} \circ \phi_{\omega_{2}} \circ \ldots \circ \phi_{\omega_{n}} .
$$

Let $\|\cdot\|$ denote the supremum norm over the set $X$. Following [4], given $t \geq 0$, we set

$$
\psi(t)=\sum_{i \in I}\left\|\phi_{i}^{\prime}\right\|^{t}
$$

and

$$
\mathrm{P}(t)=\lim _{n \rightarrow \infty} \frac{1}{n} \log \sum_{\omega \in I^{n}}\left\|\phi_{\omega}^{\prime}\right\|^{t} .
$$

The number $\mathrm{P}(t)$ is called the topological pressure of the parameter $t$. Let

$$
\theta=\theta_{S}=\inf \{t: \psi(t)<\infty\}=\inf \left\{t: \sum_{i \in I} \operatorname{diam}^{t}\left(\phi_{i}(X)\right)<\infty\right\} \geq 0
$$

and let $h_{S}=\operatorname{HD}\left(J_{S}\right)$ be the Hausdorff dimension of the limit set $J_{S}$. The following result has been proved in [4].

Theorem 3.1. It holds that

$$
\operatorname{HD}\left(J_{S}\right)=\inf \{t \geq 0: \mathrm{P}(t)<0\}=\sup \left\{h_{F}\right\} \geq \theta_{S}
$$

where the supremum is taken over all systems generated by the finite subsets of $I$.

Given $t \geq 0$ a Borel probability measure $m$ is said to be $t$-conformal provided $m\left(J_{S}\right)=1$ and for every Borel set $A \subset X$ and every $i \in I$

$$
m\left(\phi_{i}(A)\right)=\int_{A}\left|\phi_{i}^{\prime}\right|^{t} d m
$$

and

$$
m\left(\phi_{i}(X) \cap \phi_{j}(X)\right)=0,
$$

for every pair $i, j \in I, i \neq j$.

The following result has been proved in 4 .

Theorem 3.2. A t-conformal measure exists if and only if $\mathrm{P}(t)=0$ (so $t=h_{S}$ ). In addition there exists at most one t-conformal measure.

The rest of the paper is devoted to the proof of Theorem 2.7 For simplicity we will denote from now on $\Sigma=\pi\left(\mathcal{E}_{0}\right)$. The starting point of the proof is the same as in [6]; building a conformal infinite IFS (Markov partition) of the Julia-Lavaurs set that makes it look like a hyperbolic repeller. The new feature is that the [6] partition is not sufficient here. Indeed, the fact that the critical point is on the Julia-Lavaurs set if $\pi(\sigma) \in \partial \Sigma$ implies that the repeller from [6] fails to have the bounded distortion property. We must thus refine the partition, and this refinement 
depends on the phase. Here are some pictures to illustrate the situation; they show what the Julia-Lavaurs sets become as one approaches $\partial \Sigma$ along two different kinds of external rays that will appear to be the "extremals" of the possible radial approaches.

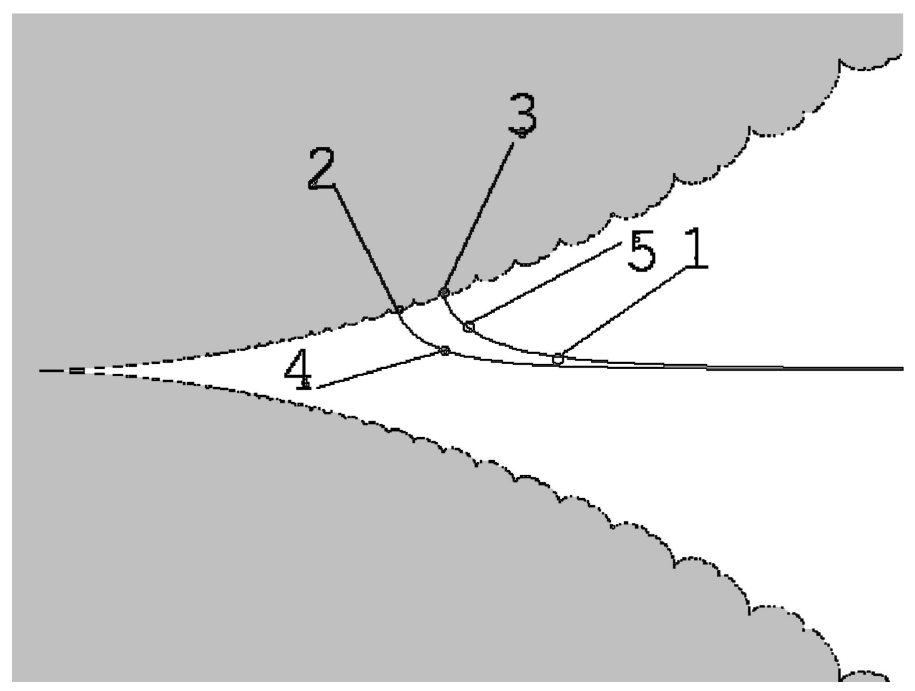

Values of the phase

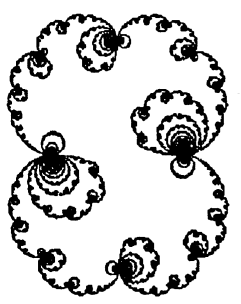

1

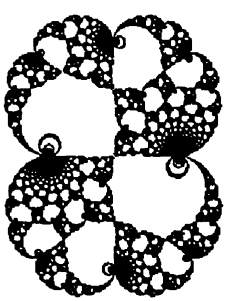

2

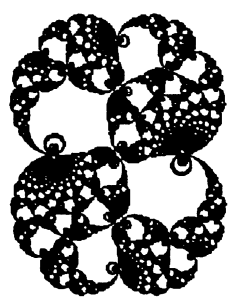

3

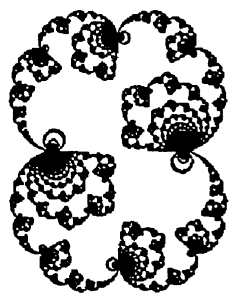

4

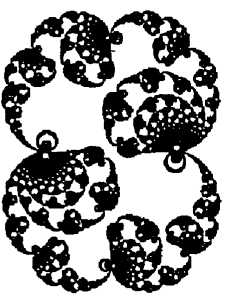

5

\section{The partition of $J\left(f_{0}, g_{\sigma}\right)$}

We start with the partition defined in [6], which will be called the DSZ partition. It is defined for Julia-Lavaurs sets corresponding to $\bar{\sigma} \in \Sigma$ in the following way: we start with $A_{0,0}$, the piece which lies between the external angles $1 / 3,2 / 3$ and define $A_{0, n}$ as being the successive preimages of $A_{0,0}$ that are in the upper half-plane and $A_{0,-n}=\bar{A}_{0, n}$. We then cut the upper wing of the butterfly at $\alpha=1 / 2$ by taking $A_{1,0}=g_{\sigma}^{-1}\left(A_{0,0}\right)$ and then $A_{1, q}=f_{0}^{q}\left(A_{1,0}\right), q \in \mathbb{Z}$; we continue on the other layers of the wing building $A_{p, q}, p \geq 0, q \in \mathbb{Z}$. Finally we do the same in the lower wing to get all the $A_{p, q}, p, q \in \mathbb{Z}$. This partition can be performed in the closed annulus $\bar{\Sigma}$ by continuity.

We now fix $\bar{\sigma} \in \partial \Sigma$ that we assume first not to correspond to a preparabolic point. We first cut $A_{0,0}$ into DSZ-cylinders of order 2 keeping all of them except the one containing $g_{\sigma}(\omega)$ and its two neighbours. We cut these three pieces into DSZ-cylinders of order 3 , keeping all of them except the one containing $g_{\sigma}(\omega)$ and 
its three neighbours, and so on. The case $g_{\sigma}(\omega)$ preparabolic is slightly different. The procedure is the same until we reach the step for which $g_{\sigma}(\omega)$ is the main cusp (the one corresponding to $\alpha$ in the cylinder). We then simply cut the cylinder into DSZ-cylinders of the next generation and stop the process. In both cases we denote by $\left\{A_{n}\right\}$ the partition of $A_{0,0}$ we have just described. Next we transfer this partition into all the DSZ-cylinders of order 1 inside the wings of the butterfly at $\alpha$, as $\left\{A_{p, q, n}, p, q \in \mathbb{Z}, p \neq 0, n \in \mathbb{N}\right\}$. Finally, putting $s(z)=-z$, we define a partition of $X=A_{0,0}$ as $\left\{B_{i}\right\}=\left\{s\left(A_{0, q}\right), q \in \mathbb{Z},|q| \geq 2\right\} \cup\left\{s\left(A_{p, q, n}\right), p \in\right.$ $\mathbb{Z}, p \neq 0, q \in \mathbb{Z}, n \in \mathbb{N}\}$. We claim that we can obtain in this way an infinite IFS. More precisely, one can construct an open simply connected neighborhood $U$ of $X$ such that for every $i$ there exists an admissible $(k, l)$ and a neighborhood $U_{i}$ of $B_{i}$ such that $g_{\sigma}^{k, l}: U_{i} \longrightarrow U$ is a conformal homeomorphism. This is due to the fact that the $B_{i}$ 's have been constructed in such a way that their diameter is small compared to their distance to the set of critical points of $g_{\sigma}^{k, l}$. The inverses of the maps $g_{\sigma}^{k, l}$ form our conformal IFS. To finish, let us mention that the "partition" we have constructed is strictly speaking not a real one since the pieces overlap. We maintain the abuse of notation since the overlap consists of no more than one point and furthermore this point will appear to be not charged by any measure we will consider. Finally, the union of the limit set of our IFS and its image by $f_{0}$ will be equal to $J\left(f_{0}, g_{\sigma}\right)$ minus the set of preparabolic points and precritical points.

\section{Determination of the $\theta$-Number of the IFS on $\partial \Sigma$}

Recall from Section 3 that the $\theta$-number of the IFS we have just constructed is the infinimum of the numbers $t>0$ such that

$$
\sum_{i} \operatorname{diam}\left(B_{i}\right)^{t}<+\infty
$$

In $\Sigma$, due to the fact that there is only one petal associated to the parabolic fixed point $\alpha$, the $\theta$-number for the DSZ-partition is equal to 1 . Since the new partition is a refinement of DSZ the series (5.1) will be convergent if and only if $t>1$ and

$$
\sum_{n} \operatorname{diam}\left(A_{1,0, n}\right)^{t}<+\infty \text {. }
$$

Since $A_{1,0, n}=g_{\sigma}^{-1}\left(A_{n}\right), \operatorname{dist}\left(g_{\sigma}(\omega), A_{n}\right) \geq c \operatorname{diam}\left(A_{n}\right)$, and since $\omega$ is a critical point of order 2 of $g_{\sigma}$, we have

$$
\operatorname{diam}\left(A_{1,0, n}\right) \approx \operatorname{dist}\left(g_{\sigma}(\omega), A_{n}\right)^{-\frac{1}{2}} \operatorname{diam}\left(A_{n}\right)
$$

for all $n$, from which it follows that the $\theta$-number of the IFS is the infinimum of the set of positive $t$ 's such that

$$
\varphi(t)=\sum_{n} \operatorname{dist}\left(g_{\sigma}(\omega), A_{n}\right)^{-\frac{t}{2}} \operatorname{diam}\left(A_{n}\right)^{t}<+\infty .
$$

Proposition 5.1. For all points on $\partial \Sigma, \theta \in[1,4 / 3]$. Moreover, $\theta=1$ if

$$
\liminf _{n \rightarrow \infty}\left|f_{0}^{n}\left(g_{\sigma}(\omega)\right)-1 / 2\right|>0
$$

while $\theta=4 / 3$ if $g_{\sigma}(\omega)$ is preparabolic.

Proof. Assume first that $g_{\sigma}(\omega)$ is not preparabolic. If we denote by $C_{n}$ the DSZcylinder of order $n$ containing $g_{\sigma}(\omega)$, then $C_{n+1}=C_{n} A_{0, k_{n}}$ for some $k_{n} \in \mathbb{Z}$ which 
indicates how close to the main cusp we are inside $C_{n}$. The contribution of the $A_{n}$ 's in (5.3) which are contained in $C_{n}$ but not in $C_{n+1}$ or its two neighbours is

$$
\varphi_{n}(t) \approx\left(\operatorname{diam} C_{n}\right)^{\frac{t}{2}} \sum_{p, q \in \mathbb{Z}}\left|\frac{1}{p+i q}-\frac{1}{k_{n}}\right|^{-\frac{t}{2}}\left(p^{2}+q^{2}\right)^{-t} .
$$

An easy computation shows that the quantity $\varphi_{n}(t) /\left(\operatorname{diam} C_{n}\right)^{t / 2}$ is of the same order as

$$
\left|k_{n}\right|^{2-\frac{3}{2} t} \int_{1}^{\left|k_{n}\right|} r^{\frac{3}{2} t-3} d r
$$

If $t>4 / 3$, then (5.4) is comparable to a constant so that

$$
\varphi(t)=\sum \varphi_{n}(t) \leq C \sum \operatorname{diam}\left(C_{n}\right)^{t / 2} \infty
$$

since $\operatorname{diam}\left(C_{n}\right)$ decreases at least as fast as a geometric series. Moreover, $\inf _{n}\left|f_{0}^{n}\left(g_{\sigma}(\omega)\right)-\alpha\right|>0 \Leftrightarrow\left(k_{n}\right) \in l^{\infty}$ and it follows that under this condition, for any $t \in] 1,4 / 3$ ], using (5.4), we have $\varphi_{n}(t) \leq C\left|k_{n}\right|^{2-3 / 2 t} \leq C^{\prime}$ if $t<4 / 3$ or $\varphi_{n}(t) \leq C \log \left|k_{n}\right| \leq C^{\prime}$ if $t=4 / 3$. Finally in the preparabolic case it is easy to see that $\varphi(t)<\infty$ if and only if

$$
\sum_{p, q \in \mathbb{Z},(p, q) \neq(0,0)}\left|\frac{1}{p+i q}\right|^{-t / 2}\left(p^{2}+q^{2}\right)^{-t}<\infty \Leftrightarrow t>4 / 3 .
$$

\section{RADIAL APPROACH}

We recall that $\bar{\sigma}_{n} \in \Sigma$ converges radially to $\bar{\sigma}_{0} \in \partial \Sigma$ if there is a lift $\sigma_{n}$ of $\bar{\sigma}_{n}$ such that $g_{\sigma_{n}}(\omega)$ converges to $g_{\sigma_{0}}(\omega)$ along an external ray of $J\left(f_{0}\right)$. Moreover, we may assume that $z_{0}=g_{\sigma_{0}}(\omega) \in A_{0,0}$. The purpose of this paragraph is to show that the external ray ending at $z_{0}$ does not pass too close to the pieces $A_{n}$.

Proposition 6.1. There exists a constant $C>0$ such that if $z$ belongs to the external ray landing at $z_{0}$, then

$$
\forall n \geq 0, \operatorname{dist}\left(z, A_{n}\right) \geq C \operatorname{diam}\left(A_{n}\right) .
$$

Proof. The property is obvious if $A_{n} \subset U_{0}$ since $\operatorname{dist}\left(A_{n}, J\left(f_{0}\right)\right) \geq c \operatorname{diam}\left(A_{n}\right)$. If $A_{n} \cap J\left(f_{0}\right) \neq \emptyset$ we argue by contradiction. Suppose there is an $n$ such that $\operatorname{dist}\left(z, A_{n}\right) \leq \epsilon \operatorname{diam}\left(A_{n}\right)$. Here there is a $k$ such that $A_{n} \subset C_{k}$ but not in $C_{k+1}$ or its two neighbours. By the bounded distortion property, applying $f_{0}$ sufficiently many times and using the fact that $f_{0}$ maps external rays on external rays, one may assume that $A_{n}=A_{0,0}$ and that the external angle is between $-\pi / 6$ and $\pi / 6$. We get an obvious contradiction if $\epsilon$ is small enough.

\section{SCHEME OF THE PROOF OF THEOREM 1}

Let $h$ be the Hausdorff dimension of $J\left(f_{0}, g_{\sigma_{0}}\right)$. Let $h_{n}$ be the Hausdorff dimension of $J\left(f_{0}, g_{\sigma_{n}}\right)$ and let $m_{n}$ be the unique $h_{n}$-conformal measure on the limit set. Passing to a subsequence, we may assume that $m_{n}$ converges weakly to a measure $m$ supported on $J\left(f_{0}, g_{\sigma}\right)$ and that $h_{n}$ converges to some number $l$. 
Lemma 7.1. $l \geq h$.

Proof. In order to prove this lemma we first observe that if two pieces of the DSZpartition intersect, the intersection consists of at most one point which cannot be charged by $m$ since it is a preperiodic repelling fixed point of $\left(f_{0}, g_{\sigma}\right)$. It then follows from [4 that $m$ satisfies (3.1). Since

$$
\sum_{|\omega|=n}\left\|\varphi_{\omega}^{\prime}\right\|^{l} \leq \sum_{|\omega|=n} K^{l} m\left(\varphi_{\omega}(X)\right) \leq K^{l}
$$

we get $\mathrm{P}(l) \leq 0$ which implies that $l \geq h$. The reasoning is now a bit weird: if $l=h$, then there is of course nothing to prove. We will then assume $l>h$, from which we will deduce the crucial formula

$$
m(\{\omega\})=0 .
$$

We know already by the results of [6] that $m(\{\alpha\})=0$. If (7.1) is true, then the measure $m$ is supported on the limit set of our IFS so that, in view of Theorem 3.2 it must be the unique such measure and $l=h$ which is a contradiction.

\section{Proof of (7.1)}

We assume that $l>h$ and we want to prove (7.1). It is enough to show that there exists a function $\psi: \mathbb{R}_{+} \longrightarrow \mathbb{R}_{+}, \psi(r) \longrightarrow 0, r \longrightarrow 0$, such that

$$
\forall n \geq 0, m_{n}(B(0, r)) \leq \psi(r) .
$$

Since $0=\omega$ is a critical point of order 2 , we may write

$$
m_{n}(B(0, r)) \leq C \sum_{k \in I(n, r)} \operatorname{dist}\left(g_{\sigma_{n}}(\omega), A_{k}\left(\sigma_{n}\right)\right)^{-h_{n} / 2} \operatorname{diam}\left(A_{k}\left(\sigma_{n}\right)\right)^{h_{n}},
$$

where $I(n, r)=\left\{k: g_{\sigma}\left(B(0, r) \cap A_{k}\left(\sigma_{n}\right) \neq \emptyset\right\}\right.$ and $A_{k}\left(\sigma_{n}\right)$ is the analogue of $A_{k}=A_{k}\left(\sigma_{0}\right)$ for $\sigma_{n}$.

Lemma 8.1. There exists $C \geq 1$ such that for every $k \geq 0$ and $n$ large enough,

$$
\operatorname{diam}\left(A_{k}\left(\sigma_{n}\right)\right) \leq C \operatorname{diam}\left(A_{k}\right), \operatorname{dist}\left(g_{\sigma_{n}}(\omega), A_{k}\left(\sigma_{n}\right)\right) \geq \frac{1}{C} \operatorname{dist}\left(g_{\sigma_{n}}(\omega), A_{k}\right) .
$$

Proof. Since the first property is invariant under iterates of $f_{0}$, we may assume that $A_{k}$ is a cylinder of order 1 of the DSZ partition. For the same reason we may even assume that

$$
g_{\sigma}^{N}\left(A_{k}\right)=A_{0,0}
$$

for some $N \geq 0$. The property then follows from the fact that

$$
\operatorname{diam}\left(A_{k}\left(\sigma_{n}\right)\right) \sim \operatorname{diam}\left(A_{k}\left(\sigma_{0}\right)\right) \sim(N+1)^{-2} .
$$

In order to prove the second property we assume first that $A_{k}$ is the cylinder of order 1 in DSZ such that $g_{\sigma}^{N}\left(A_{k}\right)=A_{0,0}$ for some $N \geq 0$. Fix $\zeta_{0}$ as the extremity of $A_{0,0}$ (i.e., $\zeta_{0}=-1 / 2 \pm i$ ) which is the furthest to $g_{\sigma_{0}}(\omega)$ and let $\zeta, \zeta_{n}$ be the corresponding points in $A_{k}\left(\sigma_{0}\right)$ and $A_{k}\left(\sigma_{n}\right)$ respectively. We claim that

$$
\left|\zeta-\zeta_{n}\right| \leq C N\left|\sigma_{0}-\sigma_{n}\right| \operatorname{diam}\left(A_{k}\left(\sigma_{0}\right)\right) \text {. }
$$


To see this inequality we write $h=\varphi^{+} \circ\left(\varphi^{-}\right)^{-1}$, the horn map. The theory of parabolic implosion shows that $h^{\prime}(Z)=1+O\left(e^{-y}\right)$. We have $g_{\sigma}^{-N}(\zeta)=\left(\varphi^{+}\right)^{-1} \circ$ $\lambda_{\sigma}^{N}\left(\varphi^{+}(\zeta)\right)$ and a short computation shows

$$
\frac{d}{d \sigma} \lambda_{\sigma}^{N}(\xi)=\left(1+\frac{d}{d \sigma} \lambda_{\sigma}^{N-1}(\xi)\right) h^{\prime}\left(\lambda_{\sigma}^{N-1}(\xi)+\sigma\right)
$$

from which (8.3) follows since $h^{\prime}\left(\lambda_{\sigma}^{N-1}(\xi)+\sigma\right)=1+\epsilon_{N}$ with $\sum\left|\epsilon_{N}\right|<\infty$. Inequality (8.3) proves the property for $N \leq K\left|\sigma_{n}-\sigma_{0}\right|^{-1}$. Indeed, recall first that $\operatorname{diam}\left(A_{k}\left(\sigma_{n}\right)\right) \leq C \operatorname{dist}\left(g_{\sigma_{n}}(\omega), A_{k}\left(\sigma_{n}\right)\right)$. Therefore we may write

$$
\operatorname{dist}\left(g_{\sigma_{n}}(\omega), A_{k}\right) \leq \operatorname{dist}\left(g_{\sigma_{n}}(\omega), A_{k}\left(\sigma_{n}\right)\right)+\operatorname{diam}\left(A_{k}\left(\sigma_{n}\right)\right)+\left|\zeta-\zeta_{n}\right|
$$

from which the result easily follows, using the first part. If $N>K\left|\sigma_{n}-\sigma_{0}\right|^{-1}$, then

$$
\operatorname{dist}\left(g_{\sigma_{n}}(\omega), A_{k}\left(\sigma_{n}\right)\right) \geq\left|g_{\sigma_{n}}(\omega)-1 / 2\right|-K^{-1}\left|g_{\sigma_{n}}(\omega)-g_{\sigma_{0}}(\omega)\right|
$$

while

$$
\operatorname{dist}\left(g_{\sigma_{n}}(\omega), A_{k}\right) \leq\left|g_{\sigma_{n}}(\omega)-1 / 2\right|+C\left|g_{\sigma_{n}}(\omega)-g_{\sigma_{0}}(\omega)\right|
$$

and we are done if $K$ is chosen big enough. During this proof we have used the formula $\left|g_{\sigma_{n}}(\omega)-g_{\sigma_{0}}(\omega)\right| \sim\left|\sigma_{n}-\sigma_{0}\right|$ and it follows from Koebe's distortion theorem and the fact that the function $\psi^{+}(\sigma)=f_{0}^{l} \circ \psi^{+}(\sigma-l)$ is univalent in a neighborhood of $\sigma_{0}$ for all $l$ large enough.

In general there is a power $f_{0}^{l}(N \in \mathbb{Z})$ mapping $A_{k}$ to an $A_{k}$ as before and it suffices to apply the preceeding to $\sigma_{0}+l, \sigma_{n}+l$. The lemma is proved.

We are led to estimate

$$
\sum_{I(n, r)} \operatorname{dist}\left(g_{\sigma_{n}}(\omega), A_{k}\right)^{-h_{n} / 2} \operatorname{diam}\left(A_{k}\right)^{h_{n}} .
$$

We split 8.4 into two parts:

The first consists of the sum over those $k$ 's for which

$$
\operatorname{dist}\left(g_{\sigma_{n}}(\omega), A_{k}\right) \geq \frac{1}{10} \operatorname{dist}\left(g_{\sigma_{0}}(\omega), A_{k}\right)
$$

which is bounded from above by the similar sum with $\sigma_{n}$ replaced by $\sigma_{0}$.

The rest consists of those $k \in I(n, r)$ that also belong to

$$
\mathcal{E}=\left\{k \in \mathbb{Z} ; \operatorname{dist}\left(g_{\sigma_{n}}(0), A_{k}\right) \leq \frac{1}{10} \operatorname{dist}\left(g_{\sigma_{0}}(0), A_{k}\right)\right\} .
$$

It follows from this definition that $k \in \mathcal{E} \Rightarrow \operatorname{dist}\left(g_{\sigma_{0}}(\omega), A_{k}\right) \leq \frac{10}{9}\left|g_{\sigma_{0}}(\omega)-g_{\sigma_{n}}(\omega)\right| \Rightarrow$ $k \in I(n, r)$ for $n$ large enough. Also $k \in \mathcal{E} \Rightarrow \operatorname{dist}\left(g_{\sigma_{n}}(\omega), A_{k}\right) \leq \frac{1}{9}\left|g_{\sigma_{n}}(\omega)-g_{\sigma_{0}}(\omega)\right|$.

Suppose that $k \in \mathcal{E}$ and let $N_{k}$ be the unique integer such that $A_{k}$ is a cylinder of order $N_{k}+1$ in $C_{N_{k}}$. An important observation is that if $k^{\prime}$ is another element of $\mathcal{E}$, then $N_{k^{\prime}}=N_{k}$.

Lemma 8.2. For $n$ large enough

$$
\sum_{k \in \mathcal{E}} \operatorname{dist}\left(g_{\sigma_{n}}(\omega), A_{k}\right)^{-\frac{h_{n}}{2}} \operatorname{diam}\left(A_{k}\right)^{h_{n}} \leq C \operatorname{diam}\left(C_{N}\right)^{\frac{h_{n}}{2}} M_{n}^{2-\frac{3}{2} h_{n}}
$$

and

$$
\sum_{k \in \mathcal{E}} \operatorname{dist}\left(g_{\sigma_{0}}(\omega), A_{k}\right)^{-\frac{h_{n}}{2}} \operatorname{diam}\left(A_{k}\right)^{h_{n}} \geq c \operatorname{diam}\left(C_{N}\right)^{\frac{h_{n}}{2}} M_{n}^{2-\frac{3}{2} h_{n}},
$$

where $N=N_{k}, M_{n}=\operatorname{diam}\left(C_{N}\right) /\left|g_{\sigma_{n}}(\omega)-g_{\sigma_{0}}(\omega)\right|$. 
Before proving this lemma, let us show why it implies the result. Indeed, in view of this lemma, if $h<h^{\prime}<l$, then for all $n$ so big that $h_{n}>h^{\prime}$, the quantity in (8.4) is bounded from above by

$$
\sum_{N_{r}}^{\infty} \operatorname{dist}\left(g_{\sigma_{0}}(\omega), A_{k}\right)^{-h^{\prime} / 2} \operatorname{diam}\left(A_{k}\right)^{h^{\prime}}
$$

where $N_{r}=\inf I(n, r) \longrightarrow \infty, r \longrightarrow 0$, and this remainder converges to 0 by (5.3) and the definition of the $\theta$-number.

In order to prove (8.5) and (8.6) we may first, by bounded distortion, assume that $N=0$ and we put $M=M_{n}$. Then

$$
\sum_{k \in \mathcal{E}} \operatorname{dist}\left(g_{\sigma_{n}}(\omega), A_{k}\right)^{-\frac{h_{n}}{2}} \operatorname{diam}\left(A_{k}\right)^{h_{n}} \leq C \sum_{\mathcal{A}_{M, N}}\left|\frac{1}{p+i q}-\frac{1}{M}\right|^{-\frac{h_{n}}{2}}\left(p^{2}+q^{2}\right)^{-h_{n}}
$$

where $\mathcal{A}_{M, N}=\left\{(p, q) \in \mathbb{Z}^{2} ;\left|\frac{1}{p+i q}-\frac{1}{N}\right| \leq \frac{1}{9 M}\right\}$. The series in this inequality is bounded above by

$$
C \iint_{|u| \leq \frac{1}{9 M}}|u|^{-\frac{h_{n}}{2}}\left|u+\frac{1}{N}\right|^{-4} d u d \bar{u} \leq C M^{2-\frac{3}{2}} h_{n}
$$

which proves (8.5). The proof of (8.6) is similar:

$$
\sum_{\mathcal{E}} \operatorname{dist}\left(g_{\sigma_{0}}(\omega), A_{k}\right)^{-\frac{h_{n}}{2}} \operatorname{diam}\left(A_{k}\right)^{\frac{h_{n}}{2}} \geq c \sum_{\mathcal{A}_{M, N}}\left|\frac{1}{p+i q}\right|^{-\frac{h_{n}}{2}+2 h_{n}} \geq c M^{2-\frac{3}{2} h_{n}}
$$

\section{REFERENCES}

[1] Adrien Douady: Does a Julia set depend continuously on the polynomial? Proceedings of Symposia in Applied Mathematics 49 (1994), 91-135. CMP 95:07

[2] Pierre Lavaurs: Systèmes dynamiques holomorphes: explosion de points périodiques paraboliques. These, Université Paris-Sud, 1989.

[3] Mariusz Urbanski and Michel Zinsmeister: Geometry of hyperbolic Julia-Lavaurs sets, Preprint 2000, to appear Indagationes Math.

[4] Dan Mauldin and Mariusz Urbanski: Dimensions and measures in infinite iterated function systems, Proc. London Math. Soc. (3) 73 (1996), 105-154. MR 97c:28020

[5] Michel Zinsmeister (after A. Douady): Basic parabolic implosion in five days. Jyvaskyla 1997.

[6] Adrien Douady, Pierrette Sentenac, and Michel Zinsmeister: Implosion parabolique et dimension de Hausdorff, C.R. Acad. Sci., Paris, Ser. I, Math. 325 (1997), 765-772. MR 98i:58195

[7] Olivier Bodart and Michel Zinsmeister: Quelques résultats sur la dimension de Hausdorff des ensembles de Julia des polynomes quadratiques. Fund. Math. (1996), 121-137. MR 97i:30034

[8] Curt McMullen: Hausdorff dimension and conformal dynamics III, Computation of dimension, Amer. J. Math. 120 (1998), 471-515. MR 2000d:37055

[9] Guillaume Havard and Michel Zinsmeister: Thermodynamic formalism and variations of the Hausdorff dimension of quadratic Julia sets, Commun. Math. Phys. 210 (2000), 225-247. CMP 2000:10 
[10] D. Ruelle: Repellors for real analytic maps, Ergodic Theory and Dyn. Sys. 2 (1982), 99-107. MR 84f:58095

[11] R. Mane, P. Sad, D. Sullivan: On the dynamics of rational maps, Ann. Scient. Ec. Norm. Sup. 16 (1983), 193-217. MR 85j:58089

Department of Mathematics, University of North Texas, P.O. Box 311430, Denton, TEXAS 76203-1430

E-mail address: urbanski@unt.edu

$U R L:$ http://www. math.unt.edu/ urbanski

Mathématiques, Université d’Orleans, BP 675945067 Orléans Cedex, France

E-mail address: Michel.Zinsmeister@labomath.univ-orleans.fr

$U R L$ : http://www.labomath.univ-orleans.fr/descriptions/zins 